\title{
Application of inverse analysis for a determination of material rheological constants basing on forming tests of circular membranes
}

\author{
I.Yu. Zakhariev ${ }^{\dagger}$, S.A. Aksenov, I.V. Logashina \\ †Ivan.Zakhariev@gmail.com
}

National Research University Higher School of Economics, 20 Myasnitskaya St., 101000, Moscow, Russia

The paper is devoted to a problem of determining rheological characteristics of materials from the results of experiments on free forming of sheet blanks to a cylindrical matrix. This information on the material behavior is used in the design of gas forming processes for the production of parts for aerospace industry. In computer simulations of the regimes for such technologies one should adequately describe the material properties. A disadvantage of the classical experimental method of tensile testing of plate specimens is that the information taken from these tests is related to metal forming in the conditions of uniaxial tension that is not typical for gas forming processes. Experimental method of forming circular membranes considered here allows one to study the behavior of a material in the conditions of biaxial tension. Interpretation of the results is proposed to carry out by an inverse analysis utilizing a special semianalytical model for the solution of the direct task. The key point of this semianalytical model is a dependence of the blank thickness at the dome pole on the dome height, which accounts for the strain rate sensitivity coefficient. This approach was applied for the processing of experimental results obtained by free forming of industrial aluminum alloys. Material characteristics obtained were compared to charecteristics determined by other methods for the same set of experimental data available in the literature. To estimate the efficiency of the method proposed, the characteristics obtained were verified by finite element simulations the results which were in good agreement with experimental data.

Keywords: inverse analysis, mathematical simulation, free bulging, rheological characteristics, aluminum alloys.

\section{Применение обратного анализа для определения реологических констант материалов по результатам тестовых формовок круглых мембран}

\begin{abstract}
Захарьев И.Ю. ${ }^{\dagger}$ Аксенов С.А., Логашина И.В.
Национальный исследовательский университет «Высшая школа экономики», ул. Мясницкая 20, 101000, Москва, Россия

Работа посвящена проблеме определения реологических характеристик материалов по результатам тестовых формовок листовой заготовки в цилиндрическую матрицу. Данная информация о материале используется при проектировании процессов газовой формовки изделий аэрокосмической отрасли. Реализация таких процессов требует соблюдения оптимальных технологических режимов, при расчетах которых необходимо адекватно описывать свойства материала, находящегося в состоянии пластического течения. Недостатком классической экспериментальной методики по растяжению плоских образцов является то, что полученная информация описывает формоизменение материала в условиях одноосного растяжения, нехарактерных для процессов газовой формовки. Рассматриваемая в работе экспериментальная методика по формовке круглых мембран позволяет исследовать поведение материала в условиях двухосного растяжения. Интерпретацию результатов тестов предлагается осуществлять посредством обратного анализа с использованием при решении прямой задачи специальной полуаналитической модели. Ключевую роль в данной полуаналитической модели играет зависимость толщины заготовки в вершине купола от высоты подъема купола, которая учитывает влияние коэффициента скоростной чувствительности. Этот подход был применен при обработке результатов испытаний по свободной формовке промышленных алюминиевых сплавов. Полученные характеристики материалов сравнивались с характеристиками, определенными с помощью других методик, для тех же наборов экспериментальных данных, доступных в литературе. С целью оценки эффективности предложенной методики, полученные константы верифицировались с помощью конечно-элементного моделирования. Результаты моделирования продемонстрировали хорошую согласованность с экспериментальными данными.
\end{abstract}

Ключевые слова: обратный анализ, математическое моделирование, газовая формовка, реологические характеристики, алюминиевые сплавы. 
Газовая формовка листовых заготовок позволяет получать корпусные изделия сложной геометрической формы, применяемые в основном в авиакосмической промышленности. Использование эффекта сверхпластичности при реализации таких процессов позволяет существенно повысить качество получаемой продукции, уменьшить количество технологических операций и снизить величину рабочего давления [1]. Технологические режимы, обеспечивающие наилучшее формоизменение уникальны для каждого изделия и рассчитываются с применением компьютерного моделирования процесса формовки [2]. При этом ключевую роль играет адекватность описания уравнений состояния материала, для определения которых проводят специальные механические испытания.

Уравнение состояния, описывающее поведение материала в условиях горячего формоизменения, представляет собой зависимость интенсивности напряжения $\sigma_{e}$ от интенсивности деформации $\varepsilon_{e}$ и скорости деформации $\dot{\varepsilon}$ :

$$
\sigma_{e}=f\left(\varepsilon_{e}, \dot{\varepsilon}_{e}\right)
$$

где $f(\ldots)$ - однозначная функция, определяемая экспериментально. Для описания реологического поведения сверхпластичных материалов может быть использована зависимость:

$$
\sigma_{e}=K \dot{\varepsilon}_{e}^{m},
$$

где $K$ и $m-$ постоянные материала.

Уравнение (2), впервые предложенное Бакофеном [3], применяется во многих исследованиях [4]. Важнейшей характеристикой в этом степенном соотношении является коэффициент скоростной чувствительности $m$, отвечающий за локализацию течения материала. Наилучшие условия пластичности достигаются при высоких значениях $m$ [5]. Считается, что сверхпластичные материалы имеют значения этой характеристики не менее 0.3 [2]. Более общий вид уравнения (2) позволяет учитывать эффект деформационного упрочнения [6]:

$$
\sigma_{e}=K \dot{\varepsilon}_{e}^{m} \varepsilon_{e}^{n},
$$

где $n$ - коэффициент деформационного упрочнения.

Классический метод определения параметров уравнения состояния материала основан на данных, полученных из эксперимента на одноосное растяжение [7]. Преимуществом данного метода является относительная простота реализации экспериментов и интерпретации результатов. Однако, в работах [8-10] показано, что параметры уравнения состояния, полученные с помощью экспериментов на одноосное растяжение, могут недостаточно хорошо описывать поведение материала в условиях двухосного растяжения, характерных для процесса формовки.

Одним из способов исследования деформационного поведения материала в условиях двухосного растяжения является формовка листовой заготовки в цилиндрическую матрицу. Интерпретация результатов таких экспериментов является нетривиальной задачей, поскольку напряжения и деформации распределены в образце неравномерно, не измеряются непосредственно в ходе эксперимента и не рассчитываются с помощью элементарных соотношений.

Методика определения реологических характеристик материала по результатам тестовых формовок листовой заготовки в цилиндрическую матрицу была впервые предложена в работе [11]. Коэффициенты $K, m$ предлагается искать следующим образом:

$$
\begin{gathered}
m=\ln \frac{p_{1} / p_{2}}{t_{2} / t_{1}}, \\
K_{i}=\frac{p_{i} R_{0}}{2 s_{0}}\left[\frac{t_{i}}{2 I_{m}(\pi / 2)}\right]^{m}, \quad i=1,2, \\
I_{m}(\alpha)=\int_{0}^{\alpha}\left(\frac{\sin ^{3} x}{x^{2}}\right)^{1 / m}\left(\frac{1}{x}-\operatorname{ctg} x\right) d x,
\end{gathered}
$$

где $t_{1}, t_{2}-$ продолжительность формовки куполов до одинаковой высоты при различных постоянных давлениях $p_{1}, p_{2}$ соответственно; $R_{0}-$ радиус матрицы; $s_{0}-$ начальная толщина заготовки.

Развитие данной методики продолжено в работах [12-14]. Работа [12] посвящена, определению коэффициентов уравнения состояния материала в виде (3) для сплава Ti-6Al-4V. Коэффициент скоростной чувствительности определялся с помощью уравнения (4), на его основании рассчитывался коэффициент деформационного упрочнения $n$. Для определения коэффициента $K$ использовались модифицированные уравнения (5), (6), учитывающие коэффициент деформационного упрочнения.

В работе [13] сравниваются результаты применения методик, описанных в работах $[11,12]$, при исследовании поведения сплава Ti-6Al-4V при температуре 1200 К. В работах [14,13] определялись характеристики сплавов AZ31(MФ2-1) и Inconel 718. Коэффициент скоростной чувствительности определялся с помощью уравнения (4), а параметры $K, n-$ с помощью обратного анализа.

Расчет значения коэффициента скоростной чувствительности $m$ с помощью уравнения (4) требует проведения тестовых формовок до одинаковой высоты купола при различных значениях давления. Для получения таких данных необходимо оборудовать формовочный пресс специальными датчиками, фиксирующими высоту заготовки в процессе формовки. В работе [16] предложена методика расчета реологических характеристик материала, не требующая одинаковой высоты заготовок.

Рассмотренные выше методики ориентированы на поиск коэффициентов уравнений (2) или (3), что исключает их применение в случаях, когда поведение материала описывается более сложными соотношениями.

Наиболее универсальным подходом, не привязанным к какой-либо конкретной форме уравнения состояния, является обратный анализ, основная идея которого заключается в многократном имитационном моделировании процесса формовки с последовательным изменением реологических характеристик материала, приводящим к значениям, обеспечивающим минимальные расхождения результатов моделирования с экспериментальными данными. В работах $[17,18]$ данный подход 
применен для исследования поведения сверхпластичных материалов ALNOVI-U и Ti-6Al-4V. Для решения прямой задачи использовался метод конечных элементов. В работе [19] предлагается использовать при обратном анализе полуаналитическую модель формоизменения купола, позволяющую существенно экономить временные и вычислительные ресурсы.

Данное исследование посвящено развитию методики, предложенной в [19], и ее применение к экспериментальным данным, опубликованным ранее $[14,19,20]$.

Рассмотрим математическую модель, описывающую формоизменение мембраны. Металлический лист с начальной толщиной $s_{0}$, формуется при постоянном давлении $P$ в цилиндрической матрицу с внутренним радиусом $R_{0}$ и входным радиусом $\rho_{0}$.

Предполагается, что свободная часть купола имеет сферическую поверхность радиуса $\rho . H-$ текущая высота купола, а $s$ - текущая толщина заготовки в вершине купола. Схема процесса формовки представлена на рис. 1.

Интенсивности деформации и скорости деформации в вершине купола определяются:

$$
\begin{gathered}
\varepsilon_{e}=\ln \left(\frac{s_{0}}{s(H)}\right), \\
\dot{\varepsilon}_{e}=-\frac{1}{s(H)} \frac{d s}{d t}=-\frac{1}{s(H)} \frac{d s}{d H} \frac{d H}{d t} .
\end{gathered}
$$

Из условия равновесия элементарного участка оболочки, расположенного в вершине купола, может быть получено уравнение для расчета интенсивности напряжения:

$$
\sigma_{e}=\frac{P \rho}{2 s(H)} .
$$

Используя соотношения (1), (7) - (9), получим дифференциальное уравнение, описывающее эволюцию высоты купола.

$$
\begin{aligned}
& \frac{d H}{d t}=-\frac{s(H)}{d s / d H} f^{-1}\left(\frac{P \rho}{2 s}, \ln \left(\frac{s_{0}}{s(H)}\right)\right), \\
& f^{-1}\left(\sigma_{e}, \varepsilon_{e}\right)=\dot{\varepsilon}_{e},
\end{aligned}
$$

где $f^{-1}(\ldots)-$ функция, обратная, к используемой в уравнении (1).

Уравнение (10) содержит функцию $s(H)$, представляющую собой зависимость толщины заготовки в вершине купола от его высоты. Для описания этой зави-

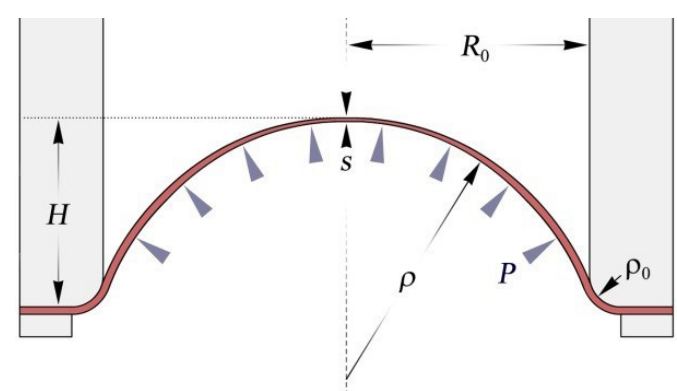

Рис. 1. Схема процесса формовки.

Fig. 1. Scheme of the free bulging process. симости предложен ряд соотношений, опирающихся на различные гипотезы о характере формоизменения оболочки в ходе формовки [11,21,22].

В работе [21] на основе гипотезы о равномерности толщины заготовки предложена зависимость:

$$
s(h)=\frac{s_{0} R_{0}^{2}}{R_{0}^{2}+h^{2}} .
$$

В работе [22], показано, что предположение о том, что в каждой точке купола выполнены условия идеального двухосного растяжения, приводит к формуле:

$$
s(h)=\frac{s_{0} R_{0}^{4}}{\left(h^{2}+R_{0}^{2}\right)^{2}} .
$$

В работе [11] принимается гипотеза о равнорастянутости меридиана, из которого следует:

$$
s(\alpha)=s_{0}\left(\frac{\sin \alpha}{\alpha}\right)^{2},
$$

где $\alpha-$ величина угла между осью симметрии и радиусом купола, проведенным к его контуру закрепления.

Допущения, принятые при построении уравнений (11) - (13) не позволяют учитывать влияние свойств материала и величину $\rho_{0}$ на величину заготовки. Для построения модели, приближенной к экспериментальным данным, в работе [19] зависимость $s(H)$ предложено искать в следующей форме:

$$
\frac{d s}{d H}=-\frac{A s^{\alpha}}{\rho},
$$

где $A$ и $\alpha-$ постоянные, которые определяются с помощью аппроксимации экспериментальных данных. Можно видеть, что при $A=1, \alpha=1, \rho_{0}=0$ уравнение (14) эквивалентно (11), а при $A=2, \alpha=1, \rho_{0}=0-(12)$.

Для определения констант $A$ и $\alpha$ в уравнении (14) необходимо иметь данные о толщине заготовки как минимум для двух различных значений высоты заготовки. В то же время, в ряде опубликованных работ, содержащих экспериментальные данные, такая информация не представлена. В работах $[14,18]$ описаны эксперименты по формовке куполов с использованием оборудования, позволяющего отслеживать высоту заготовки в процессе формовки. В результате каждого эксперимента получен массив данных, характеризующий изменение высоты купола от времени. При этом толщина заготовки измерялась один раз по окончании формовки.

Для возможности обработки результатов подобных экспериментов, зависимость $s(H)$ предлагается описывать следующим образом:

$$
s=s_{0}\left(1-B \frac{H}{\rho+\rho_{0}}\right),
$$

где $B$ - подбираемая постоянная.

Уравнение (15) может рассматриваться как обобщение зависимости $s(\mathrm{H})$, предложенной в работе [12], на случай ненулевого $\rho_{0}$. Значение константы $B$ в уравнении (15) может быть определено на основании одной пары значений высоты и толщины для каждого давления.

Подстановка уравнений (15) и (3) в (10) позволяет 
получить дифференциальное уравнение, описывающее эволюцию высоты купола:

$$
\begin{aligned}
\frac{d H}{d t}= & \frac{\rho+\rho_{0}-B H}{B H} \times(P \rho)^{1 / m} \times \\
& \times\left[2 K s_{0}\left(1-B \frac{H}{\rho+\rho_{0}}\right) \ln \left(\frac{\rho+\rho_{0}}{\rho+\rho_{0}-B H}\right)\right]^{-1 / m}
\end{aligned}
$$

Определение констант материала по результатам тестовых формовок листовой заготовки в цилиндрическую матрицу осуществлялось в два этапа. На первом этапе для каждой экспериментальной точки $\left(H_{i}, s_{i}\right)$ определялось значение $B_{i}$ :

$$
B_{i}=\frac{\left(s_{0}-s_{i}\right)\left(H_{i}^{2}-\left(R_{0}+\rho_{0}\right)^{2}\right)}{s_{0} H_{i}^{2}} .
$$

На втором этапе подбирались константы выбранного уравнения состояния путём минимизации целевой функции:

$$
F=\sum_{i=1}^{N} \min _{t}\left(\sqrt{\left(\frac{H(t)-H_{i}}{H_{i}}\right)^{2}+\left(\frac{t-t_{i}}{t_{i}}\right)^{2}}\right),
$$

где $N$ - это общее количество экспериментов, $H(t)-$ высота купола в момент времени $t$, полученная с помощью решения уравнения (16), $t_{i}$ и $H_{i}$ - экспериментальные значения времени и высоты купола.

При построении целевой функции (18) не использовалась информация о толщине заготовки, поскольку она уже была учтена на первом этапе, при вычислении значений констант $B_{i}$. Отклонение экспериментальных значений от расчетных вычислялось как минимальное расстояние от экспериментальной точки $\left(H_{i}, t_{i}\right)$ до кривой $H(t)$, полученной при решении уравнения (16).

Предложенная методика, была использована для определения параметров уравнения состояния следующих сплавов АМг6, МФ2-1 и АА5083(АМг4) по экспериментальным данным, приведенным в $[14,19,20]$.

Эксперименты для сплава АМг6 [19] проводились при постоянной температуре $415^{\circ} \mathrm{C}$ и следующих постоянных давлениях: $P_{1}=0.3, P_{2}=0.35, P_{3}=0.4, P_{4}=0.5$, $P_{5}=0.6 \mathrm{MПа.} \mathrm{Формовка} \mathrm{проводилась} \mathrm{в} \mathrm{матрицу} \mathrm{с} \mathrm{геоме-}$ трическими параметрами: $R_{0}=50 \mathrm{Mм}, \rho_{0}=5 \mathrm{mм}$. Средняя толщина используемых заготовок составляла $s_{0}=0.92 \mathrm{Mм}$.
Эксперименты для сплава МФ2-1 [14] проводились при температуре $520^{\circ} \mathrm{C}$ при постоянных давлениях $P_{1}=0.16$ и $P_{2}=0.29$ МПа. В результате экспериментов получены массивы из 36 и 19 значений высоты купола в различные моменты времени. Толщина заготовки для каждого давления измерялась один раз при максимальной высоте подъема купола. Формовка проводилась в матрицу со следующими геометрическими параметрами: $R_{0}=17.5$ мм, $\rho_{0}=3$ мм. Исходная толщина заготовок равнялась $s_{0}=0.5$ мм.

Эксперименты для сплава АМг4, описанные в работе [20], проводились при постоянной температуре $450^{\circ} \mathrm{C}$ при давлениях $P_{1}=0.29$ и $P_{2}=0.56$ МПа. При давлении $P_{1}$ было проведено 10 испытаний, при $P_{2}-8$. Испытания проводились в цилиндрическую матрицу со следующими геометрическими параметрами: $R_{0}=25 \mathrm{mм}, \rho_{0}=5 \mathrm{Mм}$, а толщина заготовок была равной $s_{0}=1.2 \mathrm{Mм}$.

С помощью предложенной методики были получены характеристики материалов. В табл. 1, для сравнения, представлены полученные характеристики и значения, опубликованные в работах $[14,19,20]$.

Несмотря на то, что коэффициент деформационного упрочнения $n$ подбирался с помощью процедуры оптимизации, его значение для сплавов АМг6 и АМг4 равно нулю с точностью до $10^{-5}$.

С целью верификации полученных результатов было проведено численное моделирование процесса формовки методом конечных элементов. Моделирование проводилось с помощью программных продуктов MSC. Patran/Nastran. Решалась 3X-мерная задача, симметричная относительно плоскостей $X Y, Z Y$. Сетка конечных элементов строилась радиально-кольцевым методом с использованием 3-х и 4-х узловых плоских элементов. Для описания материала использовались уравнение состояния вида (3). На нижнюю грань заготовки было задавалось постоянное давление, значение которого соответствовало условиям экспериментов. Пример результатов моделирования формовки сплава АМг6 представлен на рис. 2.

Сравнение полученных в результате моделирования зависимостей $H(t)$ и $s(t)$, с результатами натурных экспериментов приведено на рис. 3 и рис. 4. Экспериментальные значения показаны закрашенными маркерами,

Табл. 1. Сравнение реологических характеристик, полученных с помощью предложенной методики со значениями, опубликованными в литературе.

Table 1. Comparison between the rheological characteristics of aluminum alloys obtained by presented method and ones obtained from the literature.

\begin{tabular}{|c|c|c|c|c|c|c|c|}
\hline $\begin{array}{c}\text { Сплав } \\
\text { Alloy }\end{array}$ & \multicolumn{3}{|c|}{$\begin{array}{c}\text { Рассчитанные значения } \\
\text { Calculated parameters }\end{array}$} & \multicolumn{2}{c|}{$\begin{array}{c}\text { Значения из литературы } \\
\text { Parameters from literature }\end{array}$} & $\begin{array}{c}\text { Источник } \\
\text { Source } \\
\text { AMг6 }\end{array}$ \\
AMg6 & $\mathbf{1 6 5}$ & $\mathbf{0 . 2 7 2}$ & $\mathbf{0 . 0}$ & 155.7 & 0.275 & 0.0 & {$[19]$} \\
\hline $\begin{array}{c}\text { MФ2-1 } \\
\text { AZ31 }\end{array}$ & $\mathbf{1 5 2}$ & $\mathbf{0 . 4 7 7}$ & $\mathbf{0 . 0 4}$ & 136.6 & 0.457 & 0.013 & {$[14]$} \\
\hline AMг4 & $\mathbf{1 2 3 . 9}$ & $\mathbf{0 . 3 2 9}$ & $\mathbf{0 . 0}$ & 123.6 & 0.334 & 0.0 & {$[20]$} \\
AА5083 & & & & & & \\
\hline
\end{tabular}


данные, полученные в результате моделирования сплошными линиями. Поскольку данные о толщине заготовки сплава МФ2-1 доступны лишь на финальном этапе формовки, промежуточные значения рассчитывались с помощью уравнения (15), они отмечены на рис. 4 пустыми маркерами.

В целом, полученные при имитационном моделировании результаты хорошо согласуются с экспериментальными данными. Наибольшие отклонения наблюдаются для сплава МФ2-1. Это может быть следствием того, что уравнение Бекофена не учитывает всех особенностей поведения данного сплава в условиях горячего формоизменения.

Предложенная методика определения реологических констант материалов в условиях двухосного растяжения основана на идее обратного анализа с применением полуаналитической модели формоизменения купола
Inc: 53

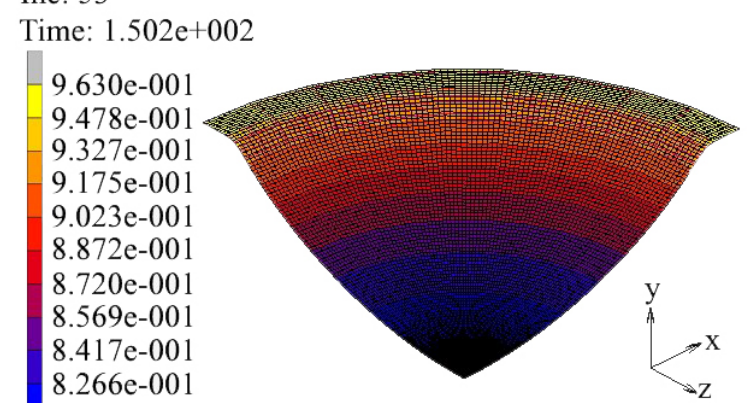

Thickness of Element

Puc. 2. (Color online) Распределение толщины заготовки сплава АМг6 на 150 секунде при формовке с постоянным давлением $0.4 \mathrm{MПа}$

Fig. 2. (Color online) Thickness distribution after 150 seconds at constant pressure of $P=0.4$ for AMg6 alloy.
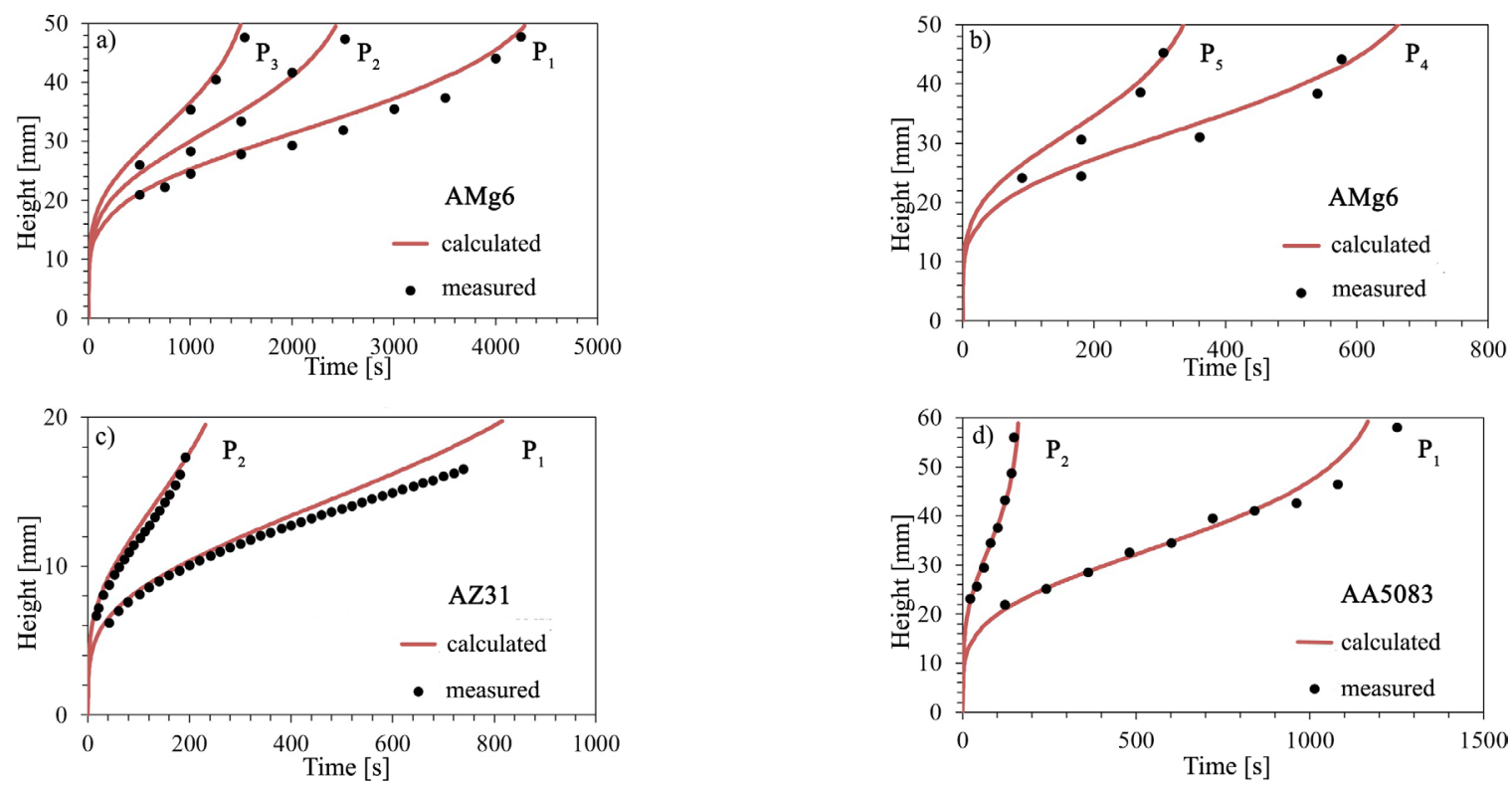

Рис. 3. Сравнение экспериментальных данных о высоте подъема купола $H(t)$ с данными компьютерного моделирования.

Fig. 3. Comparison between the dome height evolutions $H(t)$, obtained by numerical simulation and the experimental blow forming tests.
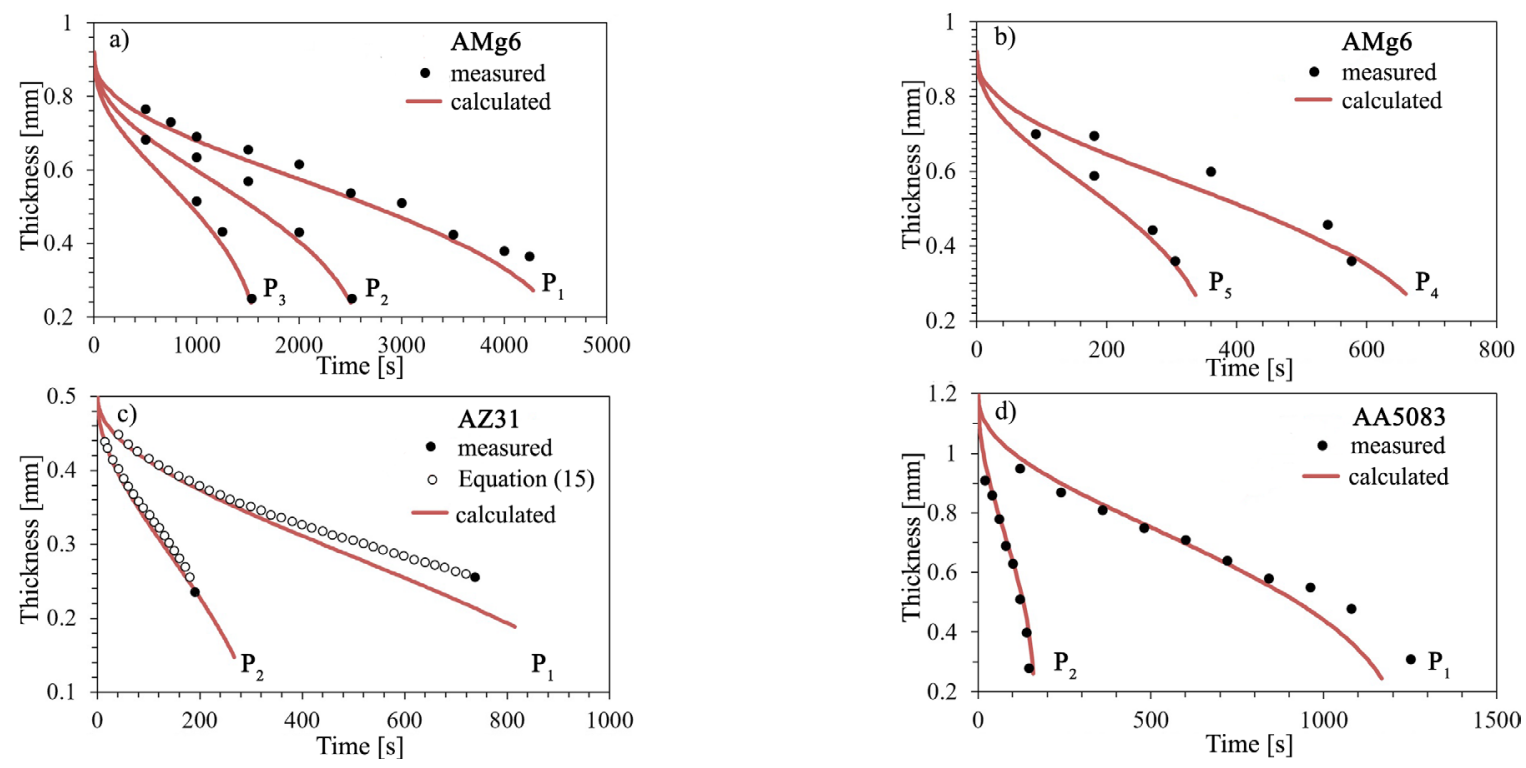

Рис. 4. Сравнение экспериментальных данных о толщине купола в его вершине $s(t)$ с данными компьютерного моделирования.

Fig. 4. Comparison between the dome thickness evolutions $s(t)$, obtained by numerical simulation and the experimental blow forming tests. 
для решения прямой задачи. Данный принцип является универсальным и позволяет использовать уравнения состояния в различной форме.

Рассчитаны константы степенного уравнения состояния, учитывающего деформационное упрочнение для сплавов АМг6, МФ2-1 и АМг4 при температурах $415^{\circ} \mathrm{C}, 520^{\circ} \mathrm{C}, 450^{\circ} \mathrm{C}$ соответственно. Построенные уравнения состояния материалов верифицировались с помощью имитационного моделирования на основе метода конечных элементов, результаты которого сравнивались с экспериментальными данными. Результаты верификации говорят об эффективности предложенной методики при интерпретации результатов испытаний по формовке листовых заготовок в цилиндрическую матрицу.

Полученные результаты могут быть использованы при проектировании технологий газовой формовки, требующих контроля режима давления для обеспечения необходимых значений скорости деформации в материале. Разработанная методика позволяет получать информацию о реологических характеристиках материала, описывающих его поведение в условиях формоизменения приближенных к реализуемым на производстве.

Благодарность/Acknowledgements. Исследование осуществлено в рамках Программы фундаментальных исследований НИУ ВШЭ в 2016 году.

\section{Литература/References}

1. E.N. Chumachenko I.V. Logashina, I.Y. Zakhariev, Metallurgist, Pages 1-6 (2016) doi:10.1007/s11015-016-0314-7 (in Russian) [Е.Н. Чумаченко, И.В. Логашина, И.Ю. Захарьев Металлург. 2016. № 4. С. $102-105$.

2. E. Chumachenko, E. Smirnov, M. Tsepin Superplasticity:Materials, theory, technology. -M. publishing house "Librikom" (2009) 320p. (in Russian) [Чумаченко Е.Н., Смирнов О.М., Цепин М.А.// М. Сверхпластичность: Материалы, теория, технологии. - М.: книжный дом “Либроком” 2009. 320с.]

3. W. A. Backofen, I. R. Turner, D. H. Avery. Trans. ASM 57 (4), pp. 980 - 990. (1964)

4. J. Hedworth, M. J. Stowell. Journal of materials science 6 pp. 1061 - 1069. (1971)

5. Hart E. W., Theory of tensile test // Acta Metall. 15, pp. $351-353 .(1967)$
6. T. Zagirov, A. Kruglov, F Enikeev. Industrial laboratory. Materials diagnostics. 9, 76, (2010) (in Russian) [Загиров Т.М., Круглов А.А., Еникеев Ф.У., “Заводская лаборатория. Диагностика материалов" № 9. Том 76 (2010)]

7. D. M. Woo. Int. J. Mech. Sci. 6, pp. $303-317$ (1964)

8. M. Albakri, F. Abu-Farha, M. Khraisheh,// Int. J. Mech. Sci. 66. pp. 55 - 66. (2013)

9. E.M. Taleff, L.G. Jr. Hector, R. Verma, P.E. Krajewski, J.K. Chang, Journal of Materials Engineering and Performance. 19 (4). pp. 488 - 494. (2010)

10. S. A. Aksenov, A.V. Kolesnikov, A.V. Mikhaylovskaya, Journal of Materials Processing Technology. 237. pp. 88 - 95. (2016) Doi:10.1016/j.jmatprotec.2016.06.003

11. F. U. Enikeev, A. A. Kruglov, Int. J. Mech. Sci. 37. pp. 473-483. (1995)

12. G. Giuliano, S. Franchitti, International Journal of Machine Tools \& Manufacture. 47. pp. 471 - 476. (2007)

13. G. Giuliano, Materials and Design. 29. pp. 1330-1333. (2008)

14. G. Giuliano, S. Franchitti, International Journal of Machine Tools \& Manufacture. 48. pp. 1519 - 1522 (2008)

15. Joon-Tae, Jong-Hoon, Ho-Sung Lee, Sung-Kie Youn, Journal of Mechanical Science and Technology. 26 (7). pp. 2101-2105 (2012)

16. F. Enikeev, O. Tulupova, V. Ganieva. Forging and Stamping Production. Material Working by Pressure 11, pp. 7-11, (2015) (in Russian) [Еникеев Ф.У., Тулупова О.П., Ганиева В.Р., «Кузнечно-штамповочное производство. Обработка материалов давлением». 11. С. 7 - 11. (2015)]

17. D. Sorgente, L. Tricarico, International Journal of Material Forming. 7. pp. $179-187$. (2014)

18. G. Y. Li, M. J. Tan, K. M. Liew, J. Mater. Process. Technol. Vol.150. pp. 76 - 83. (2004)

19. S.A. Aksenov, E.N. Chumachenko, A.V. Kolesnikov, S. A. Osipov, Journal of Materials Processing Technology. 217. pp. 158 - 164. (2015)

20. J.R. Bradley, Bulge Testing of Superplastic AA5083 Aluminum Sheet in: E.M. Taleff, P.A. Friedman, P. E. Krajewski, R. S. Mishra, J. G. Schroth (Eds.) Advances in Superplasticity and Superplastic Forming. The Minerals, Metals \& Materials Society (TMS), Charlotte, North Carolina, USA. 2004. pp. 109- 118.

21. F. Jovane, Int. J. Mech. Sci. 10. pp. 403 - 424. (1968)

22. S. Yu-Quan, Z. Jun, Mater. Sci. Eng. 84. pp. 111-125. (1986) 